\title{
Early Dural Sac Termination with Lumbar Disc Herniation: A Mimic of Nerve Root Anomalies
}

\author{
Hee-Seok Yang ${ }^{1}$, Jang-Ho Bae ${ }^{2}$, Jeong-Yoon Park ${ }^{3}$ \\ ${ }^{1}$ Department of Neurosurgery, Seoul Barunsesang Hospital, Seoul, ${ }^{2}$ Department of Neurosurgery, Jainmedi Hospital, Goyang, ${ }^{3}$ Department \\ of Neurosurgery, Spine and Spinal Cord Institute, Gangnam Severance Hospital, Yonsei University College of Medicine, Seoul, Korea
}

Corresponding Author: Jeong-Yoon Park, MD, PhD Department of Neurosurgery, Gangnam Severance Hospital, Spine and Spinal Cord Institute, Yonsei University College of Medicine, 211 Eonjuro, Gangnam-gu, Seoul 06273, Korea

Tel: $+82-2-2019-3390$

Fax: +82-2-3461-9229

E-mail: spinepjy@gmail.com

Received: July 24, 2020

Revised: August 29, 2020

Accepted: August 30, 2020
The precise location of the dural sac (DS) end is necessary for preventing neural injury during spinal surgery or procedures. There has been no report on problems with spine surgery in patients with early DS termination. A 28-year-old woman presented with low back and leg pain involving the left S1 nerve root. Magnetic resonance imaging (MRI) revealed early DS termination at the lower one-third of the $L 5$ vertebra and lumbar disc herniation at the L5/S1. Microscopic discectomy was performed instead of endoscopic discectomy to avoid unpredictable risks. Due to early DS termination, multiple nerve roots were identified, which look like nerve root congenital anomalies (Neidre and Macnab type II anomalies), and multiple separated nerve roots appeared to exit through a single foramen. After wide exposure by hemilaminectomy, which facilitated adequate visualization and mobilization of the involved nerve roots, the ruptured disc was identified and removed with gentle retraction, avoiding risk of excessive nerve root traction. Unlike other nerve root anomalies, early DS termination could be detected easily with preoperative MRI. Although this condition appears similar to other nerve root anomalies in the surgical field, it is possible to avoid inadvertent neural injury by closely investigating preoperative MRI. If early DS termination is suspected, it is necessary to consider a safer surgical approach.

Key Words: Disc herniation, Dural sac, Nerve root anomaly

\section{INTRODUCTION}

Several reports of dural sac (DS) termination anatomy using magnetic resonance imaging and cadaver examinations have been published to date, and it is generally accepted that the mean level of DS termination lies at the second sacral vertebrae ${ }^{1,6,11,12)}$. Spine surgeons are not often interested in the location of DS termination, but radiotherapists have investigated it before craniospinal irradiation for cerebrospinal (CSF) seeding and leptomeningeal metastasis, while anesthesiologists have focused on DS termination before caudal anesthesia ${ }^{5,7,16}$. A caudal epidural block is widely adopted for intraoperative and postoperative analgesia, and caudal anatomy and DS termination could have implications not only for the anesthesiologist, but also for spine surgeons for preventing neural injury during spine surgery or procedures, ${ }^{8,13)}$. Disc herniation is one of the most common diseases requiring surgical interventions, and minimally invasive discectomy with endoscopy has been increasing. During endoscopic spine surgery, unexpected anatomical anomalies are difficult to observe and address, sometimes surgeons require conversion to conventional surgery.

Lumbosacral congenital anomalies including early DS sac termination are uncommon and are encountered very rarely during spine surgery, but intraoperative nerve injury is a common complication of endoscopic lumbar discectomy with an incidence of $1.0-8.9 \%{ }^{4,18}$. If an unexpected anomaly is discovered during surgery, the possibility of complications during the operation may increase. Therefore, recognizing anomalous neural structures and early DS termination is essential to avoid iatrogenic neural injuries and to guarantee better clinical outcomes. Unfortunately, nerve root anomalies are difficult to identify during preoperative evaluations, though early DS termination may be identified early by magnetic resonance imaging (MRI) sagittal images ${ }^{15,177}$. The authors have experienced surgical treatment of the herniated lumbar disc at the L5-S1 level with early DS termination, which may be misunderstood as nerve root anomalies. As such, we herein describe our experience and review the available relevant literature. 


\section{CASE REPORT}

A 28-year-old female presented with acute low back and radiating pain in the left leg, with a positive straight-leg sign
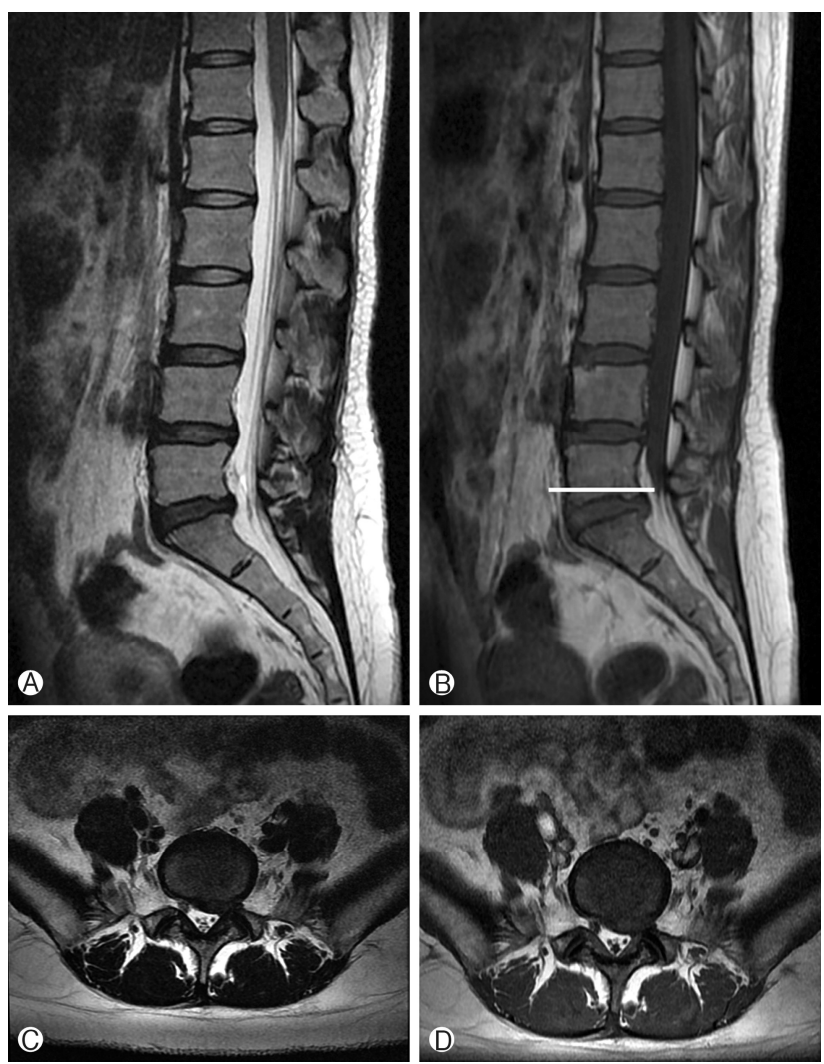

Fig. 1. MRI scans of the patient's lumbar spine. (A), (B) Sagittal T2W and T1W images demonstrate early termination of the dural sac at the lower one-third of the $L 5$ vertebrae from the point of dural sac convergence to the corresponding vertebrae (white line). (C), (D) Paramedian left herniated disc at the L5/S1 level on T2W and T1W axial MRI scans. at 30 degrees. The MRI findings show termination of the DS at the lower one-third of the L5 lumbar vertebra and herniated lumbar disc at the L5-S1 segment (Fig. 1). Due to a lack of improvement with initial conservative treatment over two months, a selective nerve root block and rehabilitation therapy were implemented. However, her symptoms had gradually worsened, and she was unable to walk due to aggravation of pain in the back and leg upon standing or walking. During a neurologic examination, S1 nerve root dermatome pain and weakness on left great toe extensor grade 3/5 were identified.

On plain lumbar radiographs, there were no anatomical anomalies. Slight disc-space narrowing at L5-S1 was noted in the lateral view. Computed tomography revealed no definite bone abnormalities. The follow-up MRI suggested aggravated disc herniation at the L5-S1 intervertebral disc level, while sagittal $\mathrm{T} 2 \mathrm{~W}$ and $\mathrm{T} 1 \mathrm{~W}$ images demonstrated early termination of the DS at the lower one-third of the L5 vertebrae (Fig. 1). Based on the above findings, we diagnosed the patient with early termination of the DS with disc herniation at L5-S1 and decided a discectomy. Recently, endoscopic discectomy for herniated lumbar disc has been popular ${ }^{3}$, but we opted to perform a conventional microscopic discectomy because of the risk of unpredictable complications due to the narrowed surgical view present during minimally invasive surgical techniques.

Intraoperatively, due to early termination of the DS, multiple nerve roots were identified (Fig. 2(A)). After wide exposure by L5 left hemilaminectomy, which enables adequate visualization and mobilization of the involved nerve roots, ruptured disc materials were identified and removed with gentle retraction, thereby avoiding the risk of laceration and excessive nerve root traction (Fig. 2(B)). While conforming to the left-side S1 pedicle medial margin, we decompressed the nerve roots sufficiently (Fig. 2(C).

\section{DISCUSSION}

Generally, the mean level of the DS termination and ending, which has been described in standard textbooks and some cadaveric studies, are located at the second sacral vertebral le-
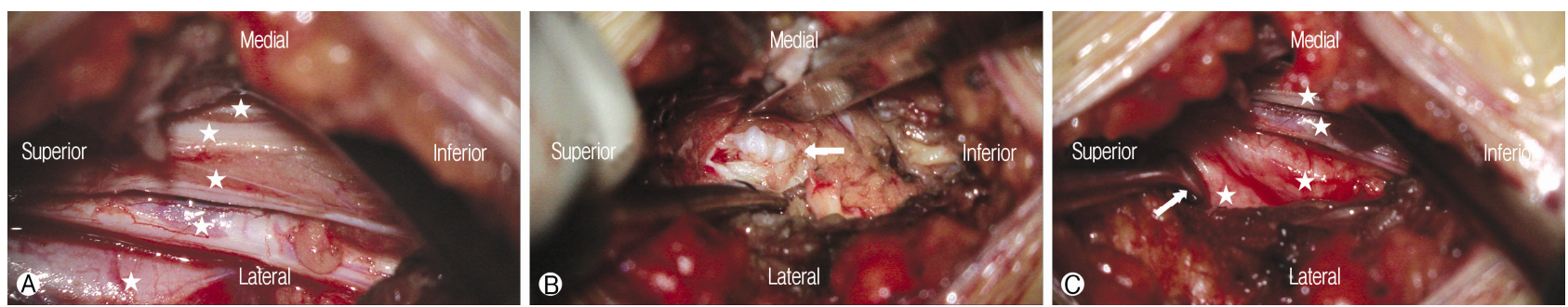

Fig. 2. Intraoperative microscopic view showing (A) multiple nerve roots (white star) after early termination of the dural sac at the lower one-third of the L5 vertebrae. (B) A wide exposure by hemilaminectomy, which facilitates adequate visualization and mobilization of the involved nerve roots, ruptured disc materials (white arrow) were identified and discectomy was done with gentle retraction, while avoiding the risk of laceration and excessive traction. (C) While conforming to the left-side S1 pedicle medial margin (white arrow), we assessed whether the nerve roots (white stars) were sufficiently decompressed. 


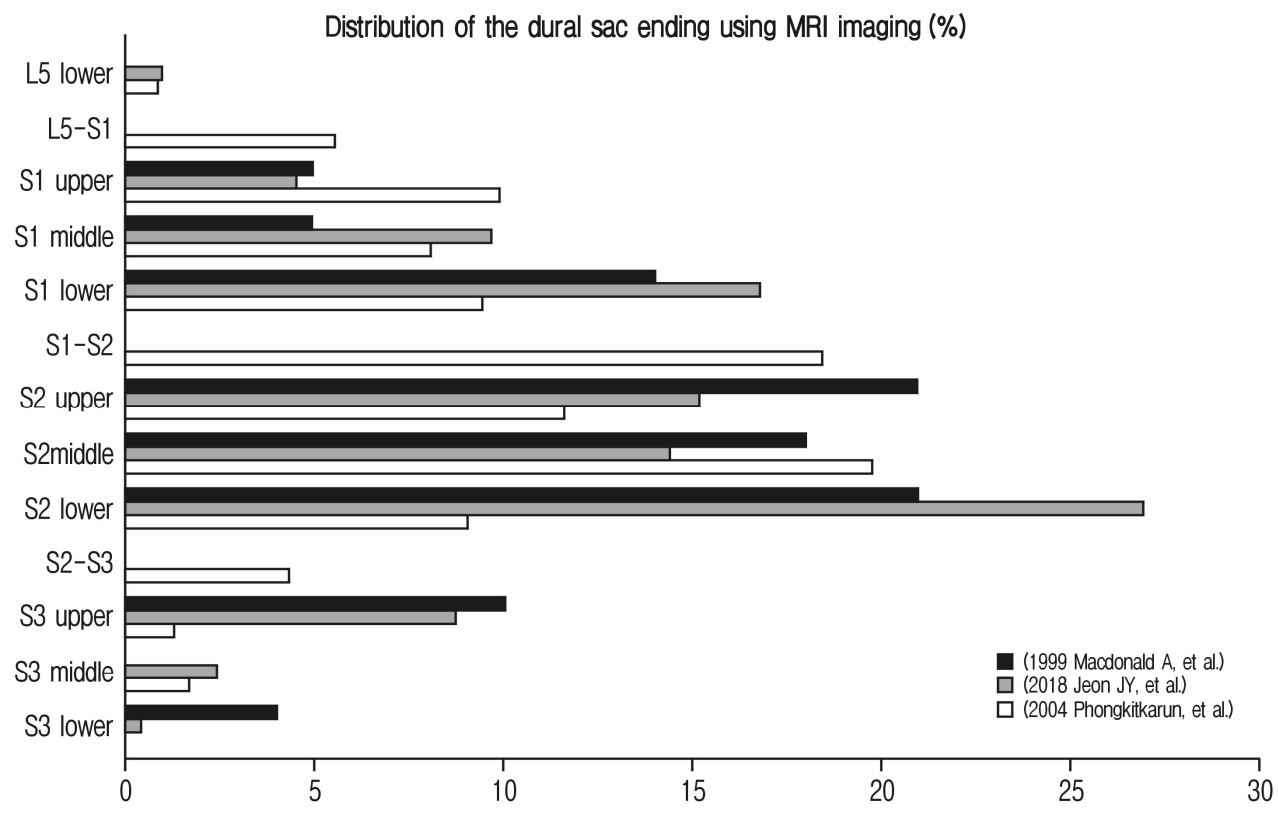

Fig. 3. Comparison of the dural sac point of termination in this case with the findings of previous studies. The frequency distribution of level of dural sac termination as determined by MRI. Three previous studies reported that the $\$ 2$ was the most frequent point of termination for the dural $\operatorname{sac}^{8,11,16)}$.

vel $^{1,6,12)}$, while the most frequent level of DS termination was at the middle one-third of S2 (Fig. 3) ${ }^{16)}$. The ending of the DS was described as the most caudal point of the DS that could be visualized on sagittal MRI. For determination of the exact level, a horizontal line was drawn from the end of dural convergence to the posterior margin of the corresponding vertebrae, and early DS termination is defined as the DS ending above the sacrum, with a reported prevalence of $0.8 \%$ to $6.4 \%$ (Fig. $3)^{6-9,11,13,16)}$. Notably, surgical problems related to early DS termination have not been reported, because it is rarer to have both early DS termination and to experience a problem requiring surgery.

In the case of CSF seeding tumors such as medulloblastoma, craniospinal irradiation is one of the treatments of choice for controlling the primary tumor and disseminated disease. Irradiation, especially that applied up to the caudal end of the CSF field, has a significant impact on treatment outcome. Therefore, precise determination of the level of DS termination during craniospinal irradiation is essential ${ }^{5,7,16)}$. Although earlier studies on the level of termination of the spinal cord and DS have been confined to cadavers and myelography, MRI has become a more accurate and convenient method by which to confirm early termination of the DS ${ }^{16)}$. A study on the caudal epidural block demonstrated a significant difference in level of DS termination on MRI scans between sacralization and lumbarization groups ${ }^{8)}$. Here, the mean caudal DS level in the lumbarization group (lower one-third of S2; 44.7\%) was significantly lower than that in the sacralization group (lower one-third of the S1; 38.8\%), and it has been reported that knowing the DS termination point could prevent injury to the dura or surrounding structures during caudal block ${ }^{8)}$.

Lumbosacral nerve root anomalies are considered uncommon congenital anomalies that are encountered rarely during spine surgeries. Surgical findings have concluded that their incidence is 1.3\%; however, cadaver studies have suggested an incidence of up to $14 \%$ in the general population, indicating that nerve root anomalies are more common than clinically reported ${ }^{9)}$. Cannon et al. classified lumbosacral nerve root anomalies into three groups according to course: conjoined roots, transverse course of the root, and anastomoses between roots ${ }^{2}$. Neidre and MacNab also classified lumbosacral anomalies ${ }^{14)}$, which is the most commonly used classification in modern clinical practice. According to the Neidre and Macnab classification scheme, type II anomalies involve redundant or "twinned" nerve roots, a situation in which two nerve roots exit through one intervertebral foramen ${ }^{14)}$. Intraoperatively, due to early termination of the DS, multiple nerve roots were identified mimicking a Neidre and Macnab type II anomaly - specifically, multiple separated nerve roots exited though one foramen (Fig. 2(A)). MRI is the gold standard for differentiating nerve root anomalies from other space-occupying pathologies. Several signs have been proposed to help in the preoperative diagnosis of nerve root anomalies, such as the sagittal shoulder sign, fat crescent sign, corner sign, and parallel sign, but these are only appropriate for type I anomalies ${ }^{10}$. Unfortunately, nerve root anomalies are difficult to identify during preoperative evaluations, although early DS termination can be identified easily in advance by closely examining MRI sagittal images ${ }^{15,17,19)}$. 
In our case, we noticed multiple nerve roots due to early DS termination combined with a herniated disc in the surgical field. This could look similar with Neidre and MacNab's type II anomaly class with multiple nerve roots. Because of the rapid advances in surgical instrumentation and techniques for minimally invasive surgery, endoscopic lumbar discectomy is gaining popularity and has become a viable alternative to conventional surgeries ${ }^{3)}$. Due to the zoom-in effect of endoscopic surgery, the advantage of seeing a very detailed image in a small space is present, but on the contrary, the overall surgical field of view is narrowed. Thus, intraoperative nerve injury is a common complication during endoscopic spinal surgery ${ }^{4,18)}$. Due to a narrow operation view, endoscopic spinal surgery may fail to detect and address nerve root anomalies properly, and if nerve roots are not properly distinguished, the probability of nerve injury will be increased. In this case, we performed microscopic discectomy instead of endoscopic discectomy to avoid unpredictable complication and risks. In addition, dilators and a working channel insertion during endoscopic spine surgery may increase the risk of iatrogenic neurologic injury, due to the abnormal nerve root anatomy of early DS termination. With sufficient exposure by conventional hemilaminectomy, which enables adequate visualization and mobilization of the involved nerve roots, ruptured disc materials were identified and removed with gentle retraction, thereby avoiding the risk of excessive nerve root traction.

\section{CONCLUSION}

Unlike other nerve root congenital anomalies, early DS termination can be detected with preoperative MRI, allowing surgeons to prepare sufficiently and safely to avoid unpredictable complication. If early DS termination is suspected, it would be desirable to choose a surgical method that can ensure a wider surgical field of view and safer surgical approach.

Conflict of Interest: The authors declare no conflict of interest.

\section{REFERENCES}

1. Berry M BL, Standring SM: Gray's anatomy. New York: Churchill Livingstone, 1995

2. Bland WC, Samuel EH, Jorge AP: Nerve-root anomalies in lumbar disc surgery. J Neurosurg 19:208-214, 1962

3. Choi G, Pophale CS, Patel B, Uniyal P: Endoscopic spine surgery. J Korean Neurosurg Soc 60:485-497, 2017

4. Choi I, Ahn JO, So WS, Lee SJ, Choi IJ, Kim H: Exiting root injury in transforaminal endoscopic discectomy: Preoperative image considerations for safety. Eur Spine J 22:2481-2487, 2013

5. Halperin EC: Impact of radiation technique upon the outcome of treatment for medulloblastoma. Int J Radiat Oncol Biol Phys 36:233-239, 1996

6. Hansasuta A, Tubbs RS, Oakes WJ: Filum terminale fusion and dural sac termination: Study in 27 cadavers. Pediatr Neurosurg 30:176-179, 1999

7. Janse van Rensburg J, Cilliers A, H. Schulenburg D: MRI determination of the vertebral termination of the dural sac tip in a South African population: Clinical significance during spinal irradiation and caudal anaesthesia. SA J Radiology 14:52-55, 2010

8. Jeon JY, Jeong YM, Lee SW, Kim JH, Choi HY, Ahn Y: The termination level of the dural sac relevant to caudal epidural block in lumbosacral transitional vertebrae: A comparison between sacralization and lumbarization groups. Pain Physician 21:73-82, 2018

9. Kadish LJ, Simmons EH: Anomalies of the lumbosacral nerve roots. An anatomical investigation and myelographic study. J Bone Joint Surg Br 66:411-416, 1984

10. Lotan R, Al-Rashdi A, Yee A, Finkelstein J: Clinical features of conjoined lumbosacral nerve roots versus lumbar intervertebral disc herniations. Eur Spine J 19:1094-1098, 2010

11. Macdonald A, Chatrath P, Spector T, Ellis H: Level of termination of the spinal cord and the dural sac: A magnetic resonance study. Clin Anat 12:149-152, 1999

12. Moore K: Clinically oriented anatomy. Baltimore: Williams \& Wilkins, 1985.

13. Nasr AY: Clinical relevance of conus medullaris and dural sac termination level with special reference to sacral hiatus apex: anatomical and MRI radiologic study. Anat Sci Int 92:456-467, 2017

14. Neidre A, MacNab I: Anomalies of the lumbosacral nerve roots. Review of 16 cases and classification. Spine 8:294-299, 1983

15. Oh CH, Park JS, Choi W-S, Choi E, Ji GY: Radiological anatomical consideration of conjoined nerve root with a case review. Anat Cell Biol 46:291-295, 2013

16. Phongkitkarun S, Jaovisidha S, Dhanachai M: Determination of the thecal sac ending using magnetic resonance imaging: Clinical applications in craniospinal irradiation. J Med Assoc Thai 87: 1368-1373, 2004

17. Shaban AB, Atiyah Q, Ajaj SS: An intraoperative finding of double L5 nerve root. Ibnosina J Med Biomed Sci 9:169, 2017

18. Tsou PM, Yeung AT: Transforaminal endoscopic decompression for radiculopathy secondary to intracanal noncontained lumbar disc herniations: Outcome and technique. The Spine J 2:41-48, 2002

19. Yu C, Zhengqi C, Xiuchun Y: An amendment to the Neidre and $\mathrm{MacNab}$ classification system for lumbosacral nerve root anomaly and its implication in percutaneous endoscopic lumbar discectomy. World Neurosurg 111:16-21, 2018 